\title{
Educación en Tiempos del Género. Consideraciones en torno a una Educación No Sexista y No Generista
}

\author{
Education in Gender Times. Considerations for a Non-Sexist \\ and Non-Genderist Education
}

\author{
Francisco Vidal Velis ${ }^{1}$ * \\ Isabel Pérez Zamora ${ }^{1}$ \\ Jaime Barrientos Delgado ${ }^{2}$ \\ Geraldine Gutiérrez Ortega ${ }^{1}$ \\ ${ }^{1}$ Universidad Metropolitana de Ciencias de la Educación, Chile \\ ${ }^{2}$ Universidad Alberto Hurtado, Chile
}

\begin{abstract}
El presente artículo analiza la forma en que el sistema educativo chileno ha reaccionado tanto a los debates socioeducativos recientes como a las transformaciones que la sociedad está experimentando en términos de género, sexualidad y familia. Los estudios realizados muestran la producción de cambios en estos ámbitos en la sociedad, no así en el sistema educacional, desde donde se invisibilizan las formas familiares alternativas, predominan visiones restrictivas del género, la sexualidad juvenil y la diversidad sexual, situaciones probablemente relacionadas con la falta de formación que los mismos profesores/as reconocen tener en estos temas. El artículo sugiere la necesidad de realizar estudios específicos con docentes que complementen el marco conceptual e investigativo con que se ha trabajado la educación no sexista con un enfoque no generista, que incorpore las disidencias sexuales, las formas familiares emergentes y otros aspectos relacionados con el género hasta ahora no considerados en las aproximaciones investigativas.
\end{abstract}

Descriptores: Educación no sexista; Educación no generista; Formas familiares alternativas; Disidencias sexuales; Inclusión.

This article analyses the way in which the Chilean educational system has reacted both to recent socio-educational debates and to the transformations that society is experiencing in terms of gender, sexuality and family. Studies show how changes have taken place in these areas in society, but not in the educational system, where alternative family forms are invisible and restrictive visions of gender, youth sexuality and sexual diversity predominate, probably due to the lack of training that teachers themselves identify in these subjects. The article suggests carrying out specific studies with teachers and complementing the conceptual and investigative framework applied to non-sexist education with a non-genderist approach, incorporating sexual dissidence, emerging family forms and other gender-related aspects, not hitherto considered in approaches to research.

Keywords: Non-sexist education; Non-genderist education; Alternative family forms; Sexual dissidence; Inclusion. 


\section{Introducción ${ }^{1}$}

A diferencia de debates anteriores que hemos tenido como sociedad -como los relativos a la ley de divorcio o aborto en tres causales- hoy día la discusión sobre género y sexualidad está teniendo profundas repercusiones en el ámbito de la educación. Entre los temas emergentes que se discuten aparece el debate sobre los colegios monogenéricos, la educación no sexista, la necesidad de legislar sobre identidad de género y matrimonio igualitario, el acoso sexual en liceos y universidades y la incorporación de personas trans en distintos niveles del sistema educativo. Sobre el primer punto, podemos recordar la discusión provocada por la carta que, en 2016, una niña envió a la expresidenta Bachelet, donde expresaba su deseo de ingresar al Instituto Nacional, emblemático establecimiento escolar chileno, exclusivo para hombres. Por otro lado, la formación de jóvenes y adolescentes en sexualidad también ha sido objeto de polémicas, esta vez suscitadas por el libro "100 preguntas sobre sexualidad adolescente" (Municipalidad de Santiago, 2016), considerado demasiado directo por algunos sectores de la sociedad, recordando las resistencias que tuvieron en su momento las Jornadas de Conversación sobre Afectividad y Sexualidad (JOCAS) (Vidal, 2002).

A ello se agrega el inusual incremento en las tasas de VIH/SIDA los últimos 5 años, sobre todo en el grupo de 19 a 29 años, incluyéndonos en la lista de los 10 países del mundo donde más crece la epidemia (ONUSIDA, 2017). Esto ha llevado a las autoridades a plantear que la educación sexual sea incorporada en las mallas curriculares desde quinto básico o incluso desde prekínder. También hubo una fuerte reacción de ciertos grupos conservadores por la distribución, en algunos establecimientos educativos, del texto del Movimiento de Liberación Homosexual "Nicolás tiene dos papás" (MOVILH, 2014), que instala la discusión sobre familias homoparentales. La apertura a formas familiares distintas de la tradicional parece ser vista como una puesta en tela de juicio de la familia nuclear biparental. Así se desprende de las consideraciones del Instituto de Estudios de la Sociedad sobre el texto aludido, donde "lo masculino y lo femenino pierden cualquier contenido objetivo, los actos sexuales tienden a perder cualquier horizonte de justificación más allá de la pura búsqueda de placer, y finalmente, la familia se convierte en un mero conjunto de personas que comparten ciertos vínculos afectivos y un mismo techo” (IES, 2014).

En 2018, hubo un fuerte debate porque un liceo de Santiago incorporó un libro del escritor homosexual chileno Pedro Lemebel como lectura recomendada para la enseñanza media. El resultado fue que los/as estudiantes se negaron a leer el texto por considerarlo "asqueroso" y, además, fueron apoyados por sus apoderados, que acusaron al profesor de querer "homosexualizar" a sus hijos. La polémica terminó con el despido del docente, ya que el director consideró que este no compartía los valores del proyecto educativo institucional. El tema abrió múltiples debates, como el rol que han adquirido los padres y apoderados en influir en la toma de decisiones pedagógicas en los establecimientos, trasgrediendo normativas legales como, en este caso, la Ley de no discriminación (Ministerio Secretaría General de Gobierno, 2012). ¿Pueden los apoderados negarse a que un establecimiento dicte clases de educación sexual o que incorpore a un estudiante trans?

\footnotetext{
${ }^{1} \mathrm{El}$ presente artículo es parte de un Proyecto más amplio de la Dirección de Investigación de la UMCE. DIUMCE FGI 11-18.
} 
Con anterioridad, en 2015, un jardín infantil se negó a llamar a una estudiante de acuerdo a su nueva identidad de género, tal como solicitaron los padres, quienes después de visitar especialistas en Chile y el extranjero, se formaron la convicción que debían respetar la identidad de género de la menor. Esta negativa los llevó a retirar a su hija del establecimiento, desde donde se señaló que no accedieron a la petición realizada por los padres, debido al efecto negativo que pudiera tener en los otros niños/as. Este año, una adolescente trans se retiró de su establecimiento educacional de origen, de varones, para inscribirse en uno de niñas, el Liceo 1. Tras el rechazo inicial del establecimiento, surgieron diversas críticas, forzando la intervención del alcalde de Santiago y de la Ministra de Educación, quienes, basándose en la ley de inclusión, concluyeron que la joven podía matricularse en el establecimiento de su preferencia.

Como vemos, todas estas discusiones tienen que ver con lo que antes correspondía al ámbito de la intimidad, considerado, por algunos autores, como el lugar donde se han producido mayores cambios en esta fase de la modernidad (Bauman, 2007; Beck, 2010; Kertzer y Barbagli, 2004; Segalen, 2013). Giddens (2003), por ejemplo, considera que ninguno de estos cambios "supera en importancia a los que tienen lugar en nuestra vida privada, en la sexualidad, las relaciones, el matrimonio y la familia” (2003, p.65). Así, el ámbito de la vida privada deja de ser privado y pasa al escrutinio público (Valdés y Valdés, 2005). Así también, las transformaciones que estarían produciéndose en la sociedad chilena, pese a corresponder a la vida privada, tienen correlato en la vida pública, pues mujeres y disidencias sexuales se hacen visibles y demandan respeto por sus derechos. En este contexto, la discusión debe darse desde la perspectiva de los Derechos Humanos, ya que estos grupos han sido excluidos de los espacios de poder por no adecuarse a las "reglas del género", que son "aquellas que protegen el marco patriarcal a través del cual mujeres y minorías sexuales y de género han sido oprimidas y controladas (Gilbert y Flem, 2011, p. 194). En un contexto de profundas transformaciones sociales, cabe preguntarse por el rol del sistema educativo en estos procesos de cambio, que terminan por incidir en lo que ocurre al interior de la sala de clases. Si un profesor/a no acepta los diversos tipos de familia de que provienen sus estudiantes, ni la forma de expresión de sus identidades sexuales y de género, probablemente adoptará actitudes excluyentes hacia estos menores, lesionando el principio de inclusividad que debiese caracterizar la acción educativa (UNESCO, 2009).

El objetivo del artículo es discutir críticamente el concepto de sexismo, la forma en que se está abordando en el campo de la investigación educativa y proponer una alternativa más amplia, tanto para su conceptualización como para su estudio en términos educacionales. El artículo consta de tres partes. La primera se centra en la discusión sobre los conceptos de sexismo, generismo y disidencia, mientras que una segunda lo hace en los cambios que la sociedad chilena experimenta en cuanto a concepciones de familia, género y sexualidad. Finalmente, se abordan los estudios que han trabajado estos temas con profesores/as de distintos niveles del sistema educacional. Se concluye con la necesidad de realizar estudios más amplios y diversos con docentes, complementando el enfoque no sexista con el no generista.

\section{Representaciones de género, sexismo y generismo}

La categoría de género permite comprender que cuestiones pensadas como atributos naturales de hombres o mujeres son, en realidad, características construidas socialmente, 
sin relación con la biología (Lamas, 2003). Esta categoría estaría constituida por "los conjuntos de prácticas, símbolos, representaciones, normas y valores sociales que las sociedades elaboran a partir de la diferencia sexual anátomo-fisiológica y que dan sentido a la satisfacción de los impulsos sexuales, a la reproducción de la especie y en general al relacionamiento entre las personas" (De Barbieri, 1993, pp.149-150). De esta manera, el género sería la construcción cultural de las diferencias sexuales (Butler, 2003) que en nuestras sociedades, se expresa en la superioridad del hombre respecto de la mujer. Para la masculinidad dominante, los hombres son "personas importantes, activas, autónomas, fuertes, potentes, cuyo ámbito de acción está en la calle, por oposición a las mujeres, a los homosexuales y a los hombres feminizados" (Olavarría, 1999, pp. 11-12). De esta manera, las representaciones de género son "elaboraciones simbólicas no sólo visuales sino también discursivas de las relaciones entre hombres y mujeres y su lugar en la sociedad" (Flores, 2007, p. 106). Sus implicancias en el ámbito educativo han comenzado a investigarse recientemente, debido al "interés cada vez mayor a identificar y eliminar tendencias sexistas en la educación” (Páramo, 2010, p. 178).

El concepto de representaciones de género tiene similitudes con el de sexismo, pero se diferencian en que las primeras, por sí mismas, no expresan un juicio de valor, es decir, pueden ser positivas o negativas, favorables o desfavorables, cambiantes o estáticas, mientras el sexismo es siempre negativo. Para Araya, el sexismo es una forma de discriminación que utiliza el sexo como criterio de atribución de cualidades, valoraciones y significados creados en la vida social, siendo posible contribuir a superarlo a través de la educación, siempre que haya un "reconocimiento de la discriminación, de la desigualdad y de la segregación como problemas educativos, pues nadie cambia aquello que no reconoce como problema" (Araya, 2004, p. 2). Por su carácter segregador, el sexismo se contrapone al ideal de igualdad que presuponen las sociedades democráticas, pero sigue estando presente en las prácticas culturales. Las consecuencias negativas del sexismo -aun cuando afectan a ambos sexos, ya que los/as limita como personas y les niega determinados comportamientos- son mayores para las mujeres, ya que las sitúa en una posición de inferioridad y de dependencia con respecto del hombre ${ }^{2}$. El sexismo puede expresarse de distintas maneras y abarcar distintos ámbitos, como la sexualidad, las relaciones laborales, las políticas salariales, los roles al interior de la familia, el cuidado de enfermos, entre otros (Moya, 2004). Sin embargo, en el ámbito que analizamos, las situaciones discriminatorias van más allá del sexo y de las relaciones de poder entre hombres y mujeres, ya que incorporan diferencias relacionadas con el género, las disidencias sexuales y las familias, por lo que creemos necesario complementar el concepto de sexismo con el de bigenerismo.

El concepto de "bigenerismo" ha sido desarrollado recientemente por Miqqui Gilbert, quien lo define como "un punto de vista que acepta las reglas del género y que no permite variaciones, excepciones o desviaciones de la regla" (Gilbert y Flem, 2011, p. 106). Quienes desafían las reglas del género habitualmente son mujeres o minorías sexuales, que reciben el rechazo de la sociedad, que puede llegar incluso a la muerte del trasgresor/a. El

\footnotetext{
${ }^{2}$ Glick y Fiske (1996) introdujeron la noción de 'sexismo ambivalente', constituido por un sexismo benevolente y uno hostil. Este último corresponde al sexismo tradicional, a la conducta discriminatoria, mientras que el benevolente se basa en una ideología que idealiza a las mujeres como esposas, madres y objetos románticos, ofreciéndoles afecto a cambio de cumplir sus roles tradicionales.
} 
bigenerismo, entonces, se convierte en la piedra angular de la heteronormatividad, punto donde convergen sexismo y bigenerismo. "El bigenerismo y la heteronormatividad aseguran que el mundo esté dividido en hombre y mujer, y el sexismo se ocupa de que la mujer sea subvalorada y el hombre sobrevalorado" (Gilbert y Flem, 2011, p. 109). El rol del bigenerismo en la creación de una cultura que rechaza la diferencia se expresa en que, "al codificar la distinción entre macho y hembra, hombre y mujer, masculino y femenino, crea una cultura violentamente sexista, heterosexista y transfóbica solo por la valoración de los sexos" (Gilbert y Flem, 2011, p. 117). Las autoras analizan tres modelos de bigenerismo, mostrándose partidarias de un cuarto, el "modelo no generista", que, a diferencia de los anteriores ${ }^{3}$, no supone distinción de género binaria ni otorga mayor valoración social a lo masculino que a lo femenino. En este modelo, "simplemente no hay aparato conceptual que nos permita definir o identificar género o sexo" (Gilbert y Flem, 2011, p. 117). Así, este modelo sería "el que se acerca al ideal de eliminar el sexismo, el heterosexismo, homofobia, transfobia y discriminación sexual” (Gilbert y Flem, 2011, p. 122).

En cuanto a educación no sexista, algunas definiciones mantienen la tensión en la diferencia biológica, desconsiderando el tema más amplio del género. Por ejemplo, Silva la entiende como "aquella que mantiene los estereotipos y roles que se le asignan culturalmente a uno u otro sexo” (Silva, 2010, p.169). A diferencia de ello, la concepción desarrollada por Nash aparece como más inclusiva y sociopolítica. Para el autor, la educación no sexista debiera "eliminar la legitimidad y reproducción de la subordinación y desigualdad, a través de la superación de los estereotipos y jerarquías de género y apuntar hacia una sociedad equitativa, con una convivencia basada en el respeto y la igualdad real, no formal, para alcanzar un desarrollo pleno de las potencialidades de cada uno de los miembros de la comunidad educativa” (Nash, 2018, p. 126)". Así, la concepción más tradicional de educación no sexista debiera complementarse con un enfoque no generista, ampliando las diferencias biológicas del sexo e incorporando el género, la sexualidad y las formas familiares contemporáneas.

En nuestra opinión, la educación sexista/bigenerista es aquella que mantiene y reproduce concepciones tradicionales de familia y de los roles que deben cumplir sus integrantes, que valora los mandatos heteronormativos del sistema sexo/género y que busca instalar y difundir visiones restrictivas de la vivencia de la sexualidad de los/as jóvenes y de grupos de la disidencia sexual. Por su parte, la educación no sexista y no generista debiera promover la igualdad de todos/as los individuos con independencia del sistema sexo/género, la participación igualitaria de todos los integrantes de la familia en los quehaceres domésticos, el rechazo a los estereotipos de género y un acercamiento más comprensivo a la sexualidad y a las identidades sexuales y de género de los individuos.

En el siguiente apartado nos referiremos a algunos cambios que experimenta la sociedad chilena en términos de concepciones de familia, representaciones de género y sexualidad, para, posteriormente, describir los estudios sobre la forma en que el profesorado visualiza estos cambios.

\footnotetext{
${ }^{3}$ Los otros modelos que se presentan son el bigenerismo estricto, el bigenerismo blando y el generismo no binario.

${ }^{4}$ Ortega (2018) habla de una "pedagogía feminista" que, aun cuando la comprende en sentido amplio, podría sentirse que excluye las masculinidades o la diversidad sexual.
} 


\section{Trasformaciones familiares, sexuales y de género}

\subsection{Transformaciones familiares}

Chile ha experimentado notables cambios en las formas de entender la familia, ya que, en décadas anteriores, el modelo nuclear biparental era el único considerado aceptable. En la actualidad, los datos muestran una diversificación en las formas de hacer familia, disminuyendo drásticamente los matrimonios, aumentando las convivencias y surgiendo con fuerza familias monoparentales, reconstituidas ${ }^{5}$, homoparentales y unipersonales, aun cuando no todas sean reconocidas como tales por las investigaciones (Ministerio de Desarrollo Social, 2015; Ministerio de Planificación, 2011; Registro Civil, 2017; Vidal, 2019). Así, aun cuando los estudios muestran que la familia predominante sigue siendo la nuclear, no sabemos qué porcentaje corresponde a familias nucleares propiamente tales y cuál a familias reconstituidas. Esto ha sido destacado por organismos internacionales, para quienes la categoría de familia nuclear en los países de Latinoamérica engloba demasiadas realidades, que pueden ser muy distintas entre sí. (Cerrutti y Binstock, 2009). Por otra parte, ningún estudio nacional revisado considera la familia homoparental, pese a la aprobación de la Ley de Acuerdo de Unión Civil (Ministerio Secretaría General de Gobierno, 2015) y al apoyo que actualmente tiene entre la población el matrimonio igualitario (Universidad Católica y GfT Adimark, 2016). Tampoco sabemos cuáles son los tipos de familia que difunden los textos escolares, que debieran mostrar a las futuras generaciones los diversos tipos de familia que existen en el país y no centrarse en un solo modelo. Asimismo, las actitudes de los chilenos/as hacia la familia también ha cambiado, evidenciándose una apertura a la píldora del día después, a la crianza de hijos por madres y padres solteras/os y un fuerte apoyo a las técnicas de reproducción asistida (Herrera y Teitelboim, 2010).

\subsection{Transformaciones sexuales y de género}

Los cambios en la sexualidad tienen mucho que ver con el género, ya que históricamente los hombres se iniciaban antes que las mujeres (Ministerio de Salud, 2000). Sin embargo, estudios recientes muestran que esto sólo es efectivo para los grupos de mayor edad, ya que, entre los/as jóvenes, la situación parece estar cambiando. Un estudio con 767 casos mostró que "la edad de la primera relación sexual es menor en todos los tramos etarios entre los hombres, sin evidenciarse un descenso en las cohortes más jóvenes, como sucede en el caso de las mujeres" (Mella et al., 2015, p. 291). Mientras las mujeres mayores de 56 años tenían una edad de iniciación de 23,93 años, el tramo de 18 a 30 años tiene una de 16,38 . Los estudios con jóvenes confirman esta transformación, concluyendo que "las edades de iniciación entre hombres y mujeres son muy similares, los primeros se iniciarían a los 16,36 años, mientras que las segundas a los 16,84 según la encuesta del Instituto Nacional de la Juventud de 2015. En cuanto a parejas sexuales, los hombres declaran mayor cantidad de parejas que las mujeres (GfT Adimark, 2017; Ministerio de Salud, 2000;), excepto en el estudio del Instituto Nacional de la Juventud, que reporta menores diferencias, con un promedio de 2,06 parejas para hombres y 1,52 para mujeres. Los

\footnotetext{
${ }^{5}$ Las familias reconstituidas son "familias formadas por matrimonios o uniones de hecho de separados y divorciados con hijos procedentes de una relación o relaciones anteriores” (Rivas, 2012 p. 30).

${ }^{6}$ Junto con proponer un interesante análisis de los usos políticos de las edades de iniciación, Palma (2013) establece una fuerte relación entre sexualidad, nivel educativo y estrato socioeconómico.
} 
varones también declaran mayor frecuencia en sus relaciones sexuales, sobre todo entre 46 y 55 años, con la media más alta, 4,10. Sin embargo, en el grupo más joven, las mujeres superan a los hombres, con medias de 3,29 y 3,23 respectivamente (Mella et al., 2015, p. 292). Algo similar ocurre con la satisfacción con la vida sexual, donde los hombres declaran mayor satisfacción en el tramo de 46 a 55 años, con un promedio de 7,7. En el grupo de menor edad, las mujeres los aventajan, con promedios de 7,90 y 7,78 respectivamente (Mella et al., 2015, p. 293)

Por otro lado, las investigaciones sobre representaciones de género también indican transformaciones, sobre todo entre los varones, pero que aún conviven con consideraciones más tradicionales. Un estudio con varones de enseñanza media mostró la existencia de una 'masculinidad semitradicional', entendida como "la mezcla y tensión de aspectos de una masculinidad tradicional arraigada en costumbres machistas, con rasgos de una masculinidad emergente o alternativa, fundada en los principios de respeto y equidad entre hombres y mujeres" (Matamala y Rodríguez, 2010, p. 77). Estos resultados son concordantes con los descritos por Valdés (2009) al analizar las representaciones de paternidad, donde, en las nuevas generaciones, aparecen aspectos subjetivos, emocionales y comunicativos que no estaban tan presentes en generaciones anteriores. El mayor involucramiento masculino en lo reproductivo afecta sus configuraciones identitarias, "que están siendo permeadas por las prácticas cotidianas de trabajo doméstico, cuidado y crianza de hijos e hijas, impactando las distintas formas en que se vive la paternidad" (Saldaña, 2018, pp. 198-199). Por otro lado, un estudio sobre adhesión a los mandatos tradicionales de género en universitarios/as encontró que las mujeres tienen menor adhesión a los mandatos masculinos comparadas con los hombres y que ambos grupos experimentan procesos de redefinición identitaria. Así, si bien los hombres son menos cuestionadores de los mandatos de género masculinos, un porcentaje se muestra crítico, así como también hay mujeres que adhieren a la masculinidad hegemónica (Mardones y Vizcarra, 2017).

\section{Sexismo en educación. La visión del profesorado}

En este apartado se abordarán las investigaciones realizadas exclusivamente con profesores/as, cuya importancia es crucial, ya que su opinión probablemente influirá en la forma de abordar los temas al interior de la clase y, subsecuentemente, con el grado de inclusión de mujeres o de estudiantes cuyas familias (o ellos mismos/as) pertenezcan a la disidencia sexual. En términos generales, la mayor parte de los estudios se centran en el sexismo, es decir, en las diferencias entre hombres y mujeres en cuanto a aptitudes o capacidades para los ámbitos científicos, por lo que la sexualidad, la familia y otros aspectos relacionados al género aparecen más desdibujados. No se sabe cuál es la opinión de los profesores/as chilenos sobre los tipos de familia que existen en el país, ni cómo se representa la familia en los textos escolares, por lo que, en estos casos, recurriremos a estudios de otros países de habla hispana. Tampoco hay una gran variedad de estudios sobre la forma en que enfrentan la sexualidad con sus estudiantes, ni cuál es la opinión que tienen respecto de los cambios que ha experimentado. Partiremos analizando los estudios sobre familia y sexualidad para, posteriormente, abordar los de sexismo en educación. 


\subsection{Estudios sobre familia, sexualidad y disidencia sexual}

Como señalamos, no hay estudios nacionales sobre representaciones de familia ni en profesores/as ni en textos escolares, por lo que usaremos dos investigaciones españolas y una colombiana. En 2008, la Universidad de Murcia analizó los tipos de familia presentes en los textos escolares, encontrando que sólo se representaban familias nucleares y monoparentales y, además, en diferentes contextos; las primeras en un ambiente hogareño, mientras que las segundas en lugares públicos (Miralles et al., 2008). Otra investigación mostró que, si bien algunos/as docentes tenían visiones exclusivamente sanguíneas y heterosexistas, la mayor parte entendía la familia como un proyecto común de vida, cuya finalidad era el crecimiento y desarrollo de sus miembros. A diferencia de ello, el establecimiento educacional analizado "reproduce una imagen uniforme y estandarizada de la familia. Esta homogeneidad puede verse reflejada en las comunicaciones dirigidas a las familias, los carteles, las solicitudes, los folletos y símbolos" (Rodríguez et al., 2018, p. 411) En Bogotá, finalmente, un estudio concluyó que aun cuando "muchos de los participantes mantienen una comprensión de la familia desde una estructura tradicional nuclear, conformada por padres e hijos, un número significativo de docentes señala que la familia es un grupo de personas unidas por una historia común que incluye lazos afectivos y de comunicación, que no necesariamente tienen un vínculo consanguíneo" (Moreno et al., 2016, p. 130).

En cuanto a la sexualidad, las investigaciones nacionales muestran que los/as docentes expresan visiones restrictivas de la sexualidad, dificultades para abordar el tema con sus estudiantes y falta de preparación en este ámbito. Un estudio en tres escuelas municipales y una particular subvencionada mostró que la mayoría de los profesores/as asocia la sexualidad con el cuerpo femenino, estableciendo una distinción entre la 'niña-dama' y la 'niña-loquita', desde donde emerge el pudor como signo de 'buena femineidad'. En el discurso de los profesores/as, la sexualidad se tematiza como un problema, un riesgo, discurso que, además, incluye otros elementos. "Por una parte, la sexualidad emerge como un signo de vulnerabilidad, incontinencia y/o peligro a vigilar, evitar o postergar. Por otra parte, el 'problema de la sexualidad' incomoda y avergüenza a los profesores. Se trata de una 'carga', un 'tema tabú' frente al cual podríamos identificar miedo a hablar, falta de lenguaje y de herramientas" (Vera, 2016, p. 10). Otro estudio destaca el recelo con que los profesores/as asumen los cambios en la sexualidad de los/as jóvenes, declarando no sentirse con las herramientas suficientes para abordar el tema con sus estudiantes, situación que se agudiza con la disidencia sexual. Trabajando con educadoras de párvulos y profesores/as de educación básica, un estudio encontró que ambos grupos consideraban que "existe un factor biológico que compone la identidad sexual. Sin embargo, la mitad de los profesores de EBA (educación básica) establecieron que el sexo biológico y el género se configuran en una relación causal inalienable, es decir, si una persona tiene un sexo definido como hombre, éste necesariamente debe ser masculino. (Julio et al., 2016 p. 65). Los autores destacan que los profesores/as creen necesaria una instancia de educación formal en el tema, ya que, en la actualidad, los conocimientos dependen de la autoformación de cada docente, reforzando la necesidad de capacitar y actualizar al profesorado en ejercicio. 
Respecto de la homosexualidad, Vera mostró falta de conocimientos y actitudes homofóbicas hacia la disidencia sexual ${ }^{7}$ en los profesores/as estudiados, para quienes "la homosexualidad no debiera mostrarse, no debiera expresarse en la escuela o al menos la hora de clases debiera ser salvaguardada de ese desorden" (Vera, 2016, p. 11). Otro estudio concluye que "existen grandes problemas en los profesores, quienes no parecen estar preparados para enfrentar el desafío de las minorías LGBT (lesbianas, gays, bisexuales, transexuales), como también entre los propios jóvenes, quienes enfrentan múltiples dificultades para asumir una orientación sexual distinta de aquello establecido como normal" (Ramírez y Contreras, 2016, p. 252). En este sentido, otras investigadoras proponen "incorporar acciones concretas en el conocimiento y enseñanza de la diversidad sexual; así como al análisis y comprensión de los procesos de inclusión/exclusión asociados a esta temática en el ámbito educativo” (Salas y Salas, 2016, p. 85).

\subsection{Estudios sobre sexismo en educación}

Los estudios muestran que los profesores/as de disciplinas científicas interactúan más con sus alumnos varones y que los refuerzan en mayor medida que a las mujeres, diferencia que se acrecienta al aumentar el nivel educacional. "Consciente o inconscientemente se tiende a valorar la importancia de la formación científica más para niños que para niñas y a explicar el éxito por la inteligencia en el caso de los niños y por el esfuerzo en el de las niñas" (Flores, 2007, p. 108). En Colombia, Páramo sostiene que las diferencias de género influyen en las evaluaciones que los/as profesores hacen de sus estudiantes y en la atribución diferencial de cualidades en función del género. Así, se confirma "la hipótesis de que las representaciones de género son un factor que incide en la manera como se atribuyen cualidades a los estudiantes por parte de los docentes" (Páramo, 2010, p. 192).

Otra investigación con 142 docentes de Santiago no encontró diferencias según el sexo/género del profesorado sobre la relación ciencia/género en educación científica, pero sí en cuanto al sexo del estudiantado. Los profesores/as "creen que el aprendizaje científico, el rendimiento académico y las habilidades dependen del sexo al que pertenece, ya que son características innatas del estudiantado" (Camacho, 2017, p. 76). Las concepciones tradicionales de la ciencia se basan "en la idea implícita que la perspectiva sexo/género no impacta la producción de conocimiento científico y, por lo tanto, no es necesario considerarla en la educación científica" (Camacho, 2017, p.77). En la misma línea, un estudio sobre habilidades matemáticas en estudiantes de kínder, madres, padres y educadoras de Santiago mostró que "tanto los adultos como los niños sostienen un estereotipo que asocia la matemática con el género masculino. Las medidas implícitas mostraron que todos los niños y las niñas de NSE (nivel socioeconómico) medio bajo asocian la matemática con lo masculino. Las niñas de NSE alto no asocian la matemática con ningún género" (Del Río et al., 2016, p. 20). Además, las educadoras "presentan un estereotipo de género que asocia la matemática al género masculino, tanto en medidas explícitas como implícitas" (Del Río et al., 2016, p. 42). En La Araucanía, otro estudio analizó los discursos de 16 profesores/as de matemáticas y de lenguaje respecto de la brecha de género en este campo, encontrando que "se identifican sesgos androcéntricos que expresan el arraigo de los estereotipos sexistas en el sistema escolar. Dichos sesgos se develan a través de los recursos lingüísticos empleados para evaluar habilidades de

\footnotetext{
${ }^{7}$ Para el concepto de homofobia, ver Barrientos, 2015.
} 
niños y niñas, factores que inciden en el desempeño y expectativas futuras" (Fernández y Hauri, 2016, p. 86).

En profesores/as universitarios, Reyes concluye que la mayoría evalua como injustas y antidemocráticas las normas de género, aun cuando un grupo minoritario estima que "son adecuadas, porque comprenden características naturales que son inherentes a cada uno de los sexos" (Reyes, 2006, p. 410). Los docentes coinciden en que la universidad "no incorpora la temática del género en sus contenidos explícitos ni como parte de los objetivos transversales del currículum" (Reyes, 2006, p. 412). En cuanto a rendimiento académico en educación superior, no se muestran diferencias significativas por género, ya que, si bien las mujeres obtienen un rendimiento superior al de los hombres, las diferencias no se mantienen al descomponer el análisis en las variables estudiadas (Paulus y Griggs, 2004).

Desde la perspectiva del currículum, Lillo analizó las lecturas sugeridas en los programas de Lenguaje y Comunicación de enseñanza media en función de la presencia o ausencia de mujeres, concluyendo que "existe una importante diferencia de género en el repertorio de programas de estudio, la cual favorece la literatura escrita por los hombres por sobre la femenina" (Lillo, 2016, p. 132). Además, los criterios de selección empleados por el Ministerio de Educación serían construidos en función del hombre, dejando en desventaja a la mujer. Así, "se puede afirmar que la literatura femenina ha sido apartada y omitida de la enseñanza de la literatura en el sistema escolar, siendo privilegiada la literatura masculina y, con ello, cánones estéticos, visiones de mundo y expresiones culturales masculinas que son legitimadas por la educación por sobre las femeninas" (Lillo, 2016, p. 135). Por otra parte, las normas de la American Psychological Association (APA), al pedir construir bibliografías con el apellido del autor/a y sólo la inicial del nombre, impide conocer el sexo de quien escribe, dificultándose un análisis de género de algún texto o programa. Se entiende que, en un determinado momento, se quiso omitir esta información, probablemente para evitar situaciones de discriminación, pero, en la actualidad, se trata justamente de lo contrario, de visibilizar el género.

\section{Conclusiones}

Algunos debates dados en el escenario nacional el último tiempo tienen que ver con cambios que pareciera experimentar la sociedad en ámbitos como el género, la sexualidad y la familia. A diferencia de debates anteriores, las controversias actuales se relacionan directamente con el ámbito educativo, como la discusión sobre colegios monogenéricos, los tipos de familia que difunden los textos escolares, las situaciones de acoso y abuso sexual en establecimientos educacionales, la incorporación de autores/as mujeres y homosexuales en los programas de enseñanza media, la normalización de la sexualidad juvenil, la necesidad de una educación sexual integral ${ }^{8}$, la demanda de una educación no sexista y la incorporación de niños/as y jóvenes trans a los distintos niveles del ciclo escolar. Estas transformaciones son refrendadas por los estudios realizados en el país, donde se evidencia la producción de cambios que afectan la familia, el género y la sexualidad. Desde el ámbito de lo familiar, pierde relevancia el modelo nuclear tradicional

\footnotetext{
${ }^{8}$ La educación sexual integral es aquella que "es capaz de desarrollar habilidades o capacidades con base en información culturalmente relevante, científicamente rigurosa y apropiada a la etapa de desarrollo en la que se encuentra la persona” (UNESCO, 2014, p. 2).
} 
que dominó el siglo XX, surgiendo otros arreglos familiares, como las familias monoparentales, reconstituidas y migrantes (Gonzálvez, 2016; Ministerio de Desarrollo Social, 2015; Olavarría, 2014; Vidal, 2019). Junto con ello, se abre la discusión sobre familias homoparentales, inseminaciones artificiales, vientres de alquiler, madres solteras por elección o mujeres que simplemente no quieren ser madres, privilegiando otros ámbitos de su vida ${ }^{9}$ (Moncó et al., 2011; Rodríguez y Martínez, 2012; Universidad Católica y GfT Adimark, 2016).

En sexualidad, las diferencias que históricamente existían entre hombres y mujeres se hacen menos marcadas, sobre todo al considerar a las nuevas generaciones sexuales, donde los sexos se equiparan en términos de edad de iniciación sexual, frecuencia de relaciones sexuales y satisfacción con la vida íntima (Mella et al., 2015; Palma, 2006). En cuanto a construcciones identitarias, los varones más jóvenes se muestran más conscientes de construir relaciones igualitarias con las mujeres, de participar en la crianza de los hijos/as, mostrarse más afectivos con ellos/as y llegar a acuerdos con sus parejas sobre las tareas domésticas (Araujo y Martucelli, 2012; Matamala y Rodríguez, 2010; Mardones y Vizcarra, 2017; Saldaña, 2018; Sharim y Rhim, 2017). En términos de opinión pública, hay un creciente apoyo a las familias homoparentales y a los derechos de las disidencias sexuales, lo que se ha expresado legislativamente en leyes que resguardan sus derechos, como la ley de no discriminación y de identidad de género (Marshall, 2018; Ministerio Secretaría General de Gobierno, 2012, 2015; Universidad Católica y GfT Adimark, 2016). Así, se constata una cierta coherencia entre los debates ocurridos en el escenario nacional con lo que vivimos como sociedad, según muestran las investigaciones disponibles.

Sin embargo, cuando analizamos el panorama desde el punto de vista educativo o desde la opinión de los profesores/as, vemos que los cambios no se han instalado con la misma rapidez o solidez. Por un lado, los estudios han abordado fundamentalmente las diferencias que observan los/as docentes entre hombres y mujeres en aptitudes para la ciencia (Camacho, 2017; Del Río y otros, 2016; Fernández y Hauri, 2016; Flores, 2007) o en el rendimiento académico (Medel y Asún, 2014; Paulus y Griggs, 2004). Al centrarse sólo en el sexismo, se dificulta discutir otros aspectos relevantes como los tipos de familia que los profesores/as consideran aceptables, los roles que deben cumplir los/as integrantes al interior de las familias, la forma en que se debieran relacionar los géneros, las actitudes hacia la sexualidad juvenil o el modo de tratar en clases la sexualidad y la disidencia sexual. Sobre estos temas hay pocos estudios en el país y, cuando los hay, los resultados no son muy halagüeños ni tranquilizadores, pues muestran una tendencia sexista en los profesores/as, para quienes la ciencia tiene más que ver con varones que con mujeres, siendo ellos el foco de la atención cuando tratan temas científicos (Flores, 2007; Julio et al., 2016; Lizana, 2008; Ramírez y Contreras, 2016; Vera, 2016).

Estudios internacionales muestran que en los establecimientos educacionales existe una sobrevaloración de las familias nucleares tradicionales por sobre otras formas familiares, por lo que menores provenientes de familias alternativas podrían quedar al margen del discurso docente, generando situaciones de menoscabo. "La homogeneidad con la que se muestra la familia interfiere en el reconocimiento de la diversidad familiar y supone una

\footnotetext{
${ }^{9}$ Para Konvalinka, las familias reconstituidas, las homoparentales, junto a las nuevas técnicas de reproducción asistida, "han abierto nuevas formas de hacer familia y han creado la necesidad de repensar el parentesco en general y la filiación en particular (2012, p. 9).
} 
situación de discriminación para los menores que no pertenecen a las llamadas familias tradicionales" (Rodríguez et al., 2018, p. 404). En sexualidad y género, el profesorado declara sentirse incómodo y sin las herramientas suficientes para abordar el tema con sus estudiantes, probablemente porque ellos/as tampoco fueron capacitados en el tema durante su formación inicial docente, situación que no parece haberse superado (Reyes, 2006). Según Ortega (2018), esto se debería a que los programas de pedagogía están al servicio del sistema imperante. "No debe sorprendernos entonces que las categorías de análisis de género, indispensable para una educación no sexista, no estén presentes en las mallas curriculares de las escuelas" (Ortega, 2018, p. 95). Así, una de las grandes deudas es la formación del profesorado en género y sexualidad en los planteles de educación superior, lo que, en nuestra opinión, requiere de estudios que complementen la perspectiva no sexista con la del no generismo. Esta complementariedad ha sido reconocida por los estudios, que indican que "la demanda por el reconocimiento de las diversas identidades y expresiones de género que superan el binomio hombre/mujer, enfrentan al cuerpo docente a una dimensión desconocida; la del trabajo con la diferencia (Martini y Bonard, 2019, p. 60). Contar con un acucioso diagnóstico del estamento docente sería un interesante insumo para planificar un programa de capacitación y entrenamiento en los temas analizados tanto en profesores/as en ejercicio como en estudiantes de pedagogía.

\section{Referencias}

Araujo, K. y Martucceli, D. (2012). Desafíos comunes. Retrato de la sociedad chilena y sus individuos. Lom Ediciones.

Araya, S. (2004). Hacia una educación no sexista. Revista electrónica Actualidades investigativas en educación, 4(2), 1-13.

Barrientos, J. (2015). Violencia homofóbica en América Latina y Chile. Ediciones el Desconcierto.

Bauman, Z. (2010). Modernidad líquida. Fondo de Cultura Económica.

Beck, U. (2010). La sociedad del riesgo. Hacia una nueva modernidad. Paidós.

Butler, J. (2003). Variaciones sobre sexo y género: Beauvoir, Wittig y Foucault. En M. Lamas (Ed.), El género. La construcción social de la diferencia sexual (pp. 303-326). PUEG.

Camacho, J. (2017). Identificación y caracterización de las creencias de docentes hombres y mujeres acerca de la relación ciencia-género en la educación científica. Estudios Pedagógicos, 43(3), 63-81. https://doi.org/10.4067/S0718-07052017000300004

Cerrutti, M. y Binstock, G. (2009). Familias latinoamericanas en transformación: Desafíos y demandas para la acción pública. Serie Políticas Sociales, 147, 37-57.

De Barbieri, T. (1993). Sobre la categoría de género. Una introducción teórico-metodológica. Debates en Sociología, 18, 145-169.

Del Río, M., Strasser, K. y Susperreguy, M. (2016). ¿Son las habilidades matemáticas un asunto de género? Los estereotipos de género acerca de las matemáticas de niños y niñas de kínder, sus familias y educadoras. Calidad en la Educación, 45, 20-53. https://doi.org/10.4067/So7 18-45652016000200002

Fernández, M. y Hauri, S. (2016). Resultados de aprendizaje en La Araucanía. La brecha de género en el SIMCE y el androcentrismo en el discurso de docentes de lenguaje y matemática. Calidad en la Educación, 45, 54-89. https://doi.org/10.4067/S07 18-45652016000200003 
Flores, R. (2007). Representaciones de género de profesores y profesoras de matemática, y su incidencia en los resultados académicos de alumnos y alumnas. Revista Iberoamericana de Educación, (43), 103-118.

Giddens, A. (2003). Un mundo desbocado. Los efectos de la globalización en nuestras vidas. Taurus.

Giddens, A. (2010). Sociología. Alianza.

GfT Adimark (2017). Primera encuesta de sexualidad. GFK Adimark.

Gilbert, M. y Flem, I. (2011). Derrotando el bigenerismo: Cambiando supuestos de género en el siglo XXI. Nomadías, 13, 103-128. https://doi.org/10.5354/0719-0905.2011.15288

Glick, P. y Fiske, S. (1996). The ambivalent sexism inventory: Differentiating hostile and benevolent sexism. Journal of Personality and Social Psychology, 7O(3), 491-512. https://doi.org/10.1037/0022-3514.70.3.491

Gonzálvez, H. (2016). Las familias transnacionales ¿una tautología? Más allá de la dicotomía distancia/proximidad geográfica. Polis, 15 (43), 511-532. https://doi.org/10.4067/So71865682016000100024

Herrera, F. y Teitelboim, B. (2010). La mirada de los chilenos a la familia. En Universidad Diego Portales (Ed.), Encuesta nacional UDP (pp. 121-135). Universidad Diego Portales.

IES. (2014). A propósito de Nicolás y sus papás.

http://www.ieschile.cl/2014/11/a-proposito-de-nicolas-y-sus-papas/

Julio, C., Kaeuffer, A., Riquelme, C., Silva, M., Osorio, M. y Torres, N. (2016). Conocimientos sobre identidad sexual de profesores y profesoras: ¿Barreras o facilitadores de construcción identitaria? Revista Latinoamericana de Educación Inclusiva, 10(2), 53-71. https://doi.org/10.4067/S0718-73782016000200005

Kertzer, D. y Barbagli, M. (2004). Historia de la familia europea. Paidós.

Konvalinka, N. (2012). Modos y maneras de hacer familia. Las familias tardias, una modalidad emergente. Siglo XXI.

Lamas, M. (2003). La antropología feminista y la categoría de género. En M. Lamas, El género. La construcción social de la diferencia sexual (pp. 303-326). PUEG.

Lillo, D. (2016). El discurso femenino omitido: la ausencia de escritoras en los programas de estudio de Lenguaje y Comunicación de enseñanza media. Nomadías, 21, 117-153.

Lizana, V. (2008). Representaciones sociales sobre feminidad de los/las estudiantes de pedagogía, en los contextos de formación docente inicial. Estudios Pedagógicos, 34(2), 115-136. https://doi.org/10.4067/S0718-07052008000200007

Mardones, K. y Vizcarra, M. (2017). Creencias de universitarios del sur de Chile sobre mandatos de género masculinos. Revista de Psicología, 26(2), 1-15. https://doi.org/10.5354/0719-0581.2017.47945

Marshall, P. (2018). Matrimonio entre personas del mismo sexo: Una aproximación desde la política del reconocimiento. Revista de Psicología, 27(4), 201-230. https://doi.org/10.4067/SO718-65682018000100201

Martínez, L. (2018). Disidencias sexuales y corporales: Articulaciones, rupturas y mutaciones. Psicoperspectivas, $17(1), 40-51$. https://doi.org/10.5027/psicoperspectivas-Vol17-Issue 1-fulltext-1141

Martini, G. y Bornand, M. (2019). Hacia una educación no sexista: Tensiones y reflexiones desde la experiencia de escuelas en transformación. Nomadías, 26, 45-67. 
Matamala, M. y Rodríguez, M. (2010). Estudio exploratorio sobre la identidad de género de hombres adolescentes pertenecientes al sector barrio norte de Concepción. Última Década, 18(33), 61-84. https://doi.org/10.4067/So7 18-22362010000200005

Medel, R. y Asún, R. (2014). Encuestas de evaluación docente y sesgos de género: Un estudio exploratorio. Calidad en la Educación, 40, 171-199. https://doi.org/10.4067/So718-45652014000100006

Mella, C., Oyanedel, J., Vargas, S. y De Ugarte, N. (2015). Salud sexual en Chile: Una aproximación descriptiva al comportamiento y la satisfacción sexual de los chilenos. Revista Chilena de Obstetricia y Ginecología, 80(4), 289-296. https://doi.org/10.4067/S0717-75262015000400003

Ministerio de Desarrollo Social. (2015). Encuesta de caracterización socioeconómica CASEN 2015. Ministerio de Desarrollo Social.

Ministerio de Planificación. (2011). Mujer y familia CASEN 2011. Ministerio de Planificación.

Ministerio de Salud. (2000). Estudio nacional de comportamiento sexual. Ministerio de Salud.

Ministerio Secretaría General de Gobierno. (2012). Medidas contra la discriminación. Ministerio Secretaría General de Gobierno.

Ministerio Secretaría General de Gobierno. (2015). Crea el acuerdo de unión civil. Ministerio Secretaría General de Gobierno.

Miralles, P., Delgado, C. y Caballero, M. (2008). Análisis del concepto de familia en las imágenes de los libros de texto de educación infantil. Enseñanza de las Ciencias Sociales, 7, 89-98.

Moncó, B., Jociles, M. y Rivas, A. (2011). Madres solteras por elección: Representaciones sociales y modelos de legitimación. Nueva Antropología, 24(4), 73-92.

Moreno, I., Bermúdez, A., Mora, C., Torres, D. y Ramos, J. (2016). Representaciones sociales de los maestros sobre la familia y su rol en la escuela. Revista Encuentros, 14(1), 119-138.

MOVIH. (2014). Nicolás tiene dos papás. http://www.movilh.cl/documentacion/Nicolas\%20tiene\%20dos\%20pap\%C3\%A 1s.pdf

Moya, M. (2004). Actitudes sexistas y nuevas formas de sexismo. En E. Barberá y I. Martínez (Coords.), Psicología y género (pp. 271-294). Pearson Educación.

Municipalidad de Santiago. (2016). 100 preguntas sobre sexualidad adolescente. Municipalidad de Santiago.

Nash, C. (2018). Educación no sexista y disidencia. Una mirada desde los derechos humanos. Nomadías, 25, 123-134.

Olavarría, J. (1999). De la identidad a la política: masculinidades y políticas públicas. Auge y ocaso de la familia nuclear patriarcal en el siglo XX. En J. Olavarría y R. Parrini (Eds.), Masculinidades. Identidad, sexualidad y familia (pp. 11-28FLACSO-Chile. h

Olavarría, J. (2014). Transformaciones de la familia conyugal en Chile en el período de la transición a la democracia (1990-2011). Polis, 13(37), 473-497. https://doi.org/10.4067/S0718-65682014000100025

ONUSIDA. (2017). Estimaciones de VIH y SIDA. ONU.

Ortega, E. (2018). Señas para una educación feminista. Nomadías, 25, 91-100.

Palma, I. (2006). Sociedad chilena en cambio, sexualidades en transformación (Tesis de doctorado). Universidad de Chile, Chile. 
Palma, I. (2013). Sexualidad, moral y cuentas falsas. Usos políticos de la edad de la primera relación sexual en Chile. Nomadías, 17, 133-157. https://doi.org/10.5354/0719-0905.2013.29942

Páramo, P. (2010). Las representaciones de género en profesores universitarios. Estudios Pedagógicos, 36(2), 177-193. https://doi.org/10.4067/S0718-07052010000200010

Paulus, N. y Griggs, T. (2004). Proceso de examinación del CSE: Incidencia de la variable género en el rendimiento académico. Calidad en la Educación, 21, 239-269. https://doi.org/10.31619/caledu.n21.334

Ramírez M. y Contreras, S. (2016). Narrativas de identidad afectivo-sexual LGTB en contextos escolares: El aparecer frente al Otro. Estudios Pedagógicos, 42(1), 235-254. https://doi.org/10.4067/S0718-07052016000100015

Registro Civil. (2017). Datos registrales con enfoque de género. Registro Civil.

Reyes, J. (2006). Trabajadoras(es) de la educación superior y reproducciones de género. Calidad en la Educación, (24), 397-422. https://doi.org/10.31619/caledu.n24.284

Rivas, A. (2012). El ejercicio de la parentalidad en las familias reconstituidas. Portularia, 12(2), 2941. https://doi.org/10.5218/prts.2012.0042

Rodríguez, C. y Martínez, K. (2012). El contrato de maternidad subrogada: La experiencia estadounidense. Revista de Derecho, 25(2), 59-81.

https://doi.org/10.4067/S0718-09502012000200003

Rodríguez, J., Moreno, E. y Montérdez, I. (2018). Un estudio de caso sobre la idealización y representación de la familia en los centros educativos. Estudios Pedagógicos, 44(1), 403-413. https://doi.org/10.4067/S0718-07052018000100403

Salas, N. y Salas, M. (2016). Tiza de colores: Hacia la enseñanza de la inclusión sobre diversidad sexual en la formación inicial docente. Revista Latinoamericana de Educación Inclusiva, 10(2), 73-91. https://doi.org/10.4067/S0718-73782016000200006

Saldaña, L. (2018) Relaciones de género y arreglos domésticos: Masculinidades cambiantes en Concepción, Chile. Polis, $17(50)$, 183-204. https://doi.org/10.4067/SO718-65682018000200183

Salinas, H. (2008). Políticas de disidencia sexual en México. CONAPRED.

Segalen, M. (2013). Sociología de la familia. Editorial de la Universidad Nacional de Mar del Plata.

Sharim, D. y Rihm, A. (2017) Desigualdades de género: El conflicto en las relaciones de intimidad. Centro de Estudios de Conflicto y Cohesión Social.

Silva, I. (2010). Repensando la escuela desde la coeducación. una mirada desde Chile. Revista Venezolana de Estudios de la Mujer, 15(34), 161-176.

UNESCO. (2009). La educación inclusiva: El camino hacia el futuro. Oficina Internacional de Educación.

UNESCO. (2014). Educación integral de la sexualidad: Conceptos, enfoques y competencias. UNESCO/OREALC.

Universidad Católica y GfT ADIMARK (2016). Encuesta nacional bicentenario. Universidad Católica y GfT ADIMARK.

Valdés, T. y Valdés, X. (2005). Familia y vida privada. Transformaciones, tensiones, resistencias y nuevos sentidos. FLACSO-Chile.

Valdés, X. (2009). El lugar que habita el padre en el Chile contemporáneo. Estudio de las representaciones sobre la paternidad en distintos grupos sociales. Polis, 8(23), 385-410. https://doi.org/10.4067/S0718-65682009000200017 
Vera, A. (2016). La sexualidad en los discursos de profesores de escuelas públicas de Santiago: Convivencias religioso-estatales. En L. Bahamondes y N. Marín (Eds.), Religión y espacio público (pp. 151-176). CRANN.

Vidal, F. (2002). Sexualidad y modernidad en Chile: Una relación espúria. En F. Vidal y C. Donoso. (Eds.), Cuerpo y sexualidad (pp. 27-44). FLACSO-Chile.

Vidal, F. (2017). Transformaciones familiares en Chile: Modelos familiares emergentes. Miles Chile.

Vidal, F. (2019). Familia, género y sexualidad en las teleseries chilenas contemporáneas. Afinidades con la teoría sociológica y los estudios realizados en el país. Polis, 18(53), 310-328.

\section{Breve CV de los/as autores/as}

\section{Francisco Vidal Velis}

Doctor en Sociología de la Universidad Alberto Hurtado, becario Programa de formación del capital humano avanzado de CONICYT, 2010-2014. Es profesor titular del Departamento de Formación Pedagógica de la Universidad Metropolitana de Ciencias de la Educación, ha participado en diversos congresos nacionales e internacionales en los temas que ha trabajado desde sus inicios, como sexualidad, género, diversidad y VIH/SIDA. Es autor de varios libros y de publicaciones científicas en sus áreas de interés. ORCID ID: https://orcid.org/0000-0003-4214-219X. Email: vidalvelis@yahoo.com

\section{Isabel Pérez Zamora}

Doctora en Estudios Culturales y Sociología de la Universidad de Birmingham, Inglaterra, con Beca Presidente de la República. Es profesora asociada del Departamento de Formación Pedagógica de la Universidad Metropolitana de Ciencias de la Educación y docente de pregrado y postgrado en Universidades chilenas y extranjeras. Ha colaborado en varios proyectos FONDECYT y se ha especializado en el tema de la ciudadanía sexual, participando como conferencista en distintos congresos europeos. ORCID ID: https://orcid.org/0000-0003-2076-6807. Email: isabel.perez_z@umce.cl

\section{Jaime Barrientos Delgado}

Doctor en Psicología Social de la Universitat de Barcelona, con dos estadías postdoctorales en el Institut d'Etudes Demographiques, Francia y la Universitat de Barcelona. Es profesor de la Facultad de Psicología de la Universidad Alberto Hurtado y ha ejecutado proyectos FONDECYT desde 2007. Es investigador asociado del Laboratorio Interdisciplinario de Subjetividad y Cambio Social (UAH-PUC) y del proyecto Homosexualities: knowledge and methods del Institut d'Etudes Demographiques, Francia. ORCID ID: https://orcid.org/0000-0001-8497-3552. Email: jbarrientos@uahurtado.cl

\section{Geraldine Gutiérrez Ortega}

Socióloga de la Universidad de Chile, con especialización en análisis de datos estadísticos de la Universidad Católica de Chile. Es académica del Departamento de Formación Pedagógica de la Universidad Metropolitana de Ciencias de la Educación, analista de la Unidad de Mediación en el Consejo de Defensa del Estado e investigadora asociada de DCK Research, desde donde ha estado a cargo de la ejecución de diversos estudios. También ha participado en investigaciones sobre el tema de la sexualidad y el VIH/SIDA. 
Revista Latinoamericana de Educación Inclusiva

ORCID ID: https://orcid.org/0000-0003-3442-3477. Email: gutierrez.geraldine@gmail.com 